\title{
Organic J-Aggregate Nanodots with Enhanced Light Absorption and Near-Unity Fluorescence Quantum Yield
}

\author{
Hubert Piwoński,* Shuho Nozue, Hiroyuki Fujita, Tsuyoshi Michinobu, and Satoshi Habuchi* \\ Cite This: https://doi.org/10.1021/acs.nanolett.0c04928 \\ Read Online
}

ABSTRACT: Development of biocompatible fluorophores with small size, bright fluorescence, and narrow spectrum translate directly into major advances in fluorescence imaging and related techniques. Here, we discover that a small donor-acceptordonor-type organic molecule consisting of a carbazole $(\mathrm{Cz})$ donor and benzothiazole (BT) acceptor ( $\mathrm{CzBTCz}$ ) assembles into quasicrystalline J-aggregates upon a formation of ultrasmall nanoparticles. The $3.5 \mathrm{~nm} \mathrm{CzBTCz}$ Jdots show a narrow absorption spectrum ( fwhm $=27 \mathrm{~nm}$ ), near-unity fluorescence quantum yield $\left(\phi_{\mathrm{fl}}=0.95\right)$, and enhanced peak molar extinction coefficient. The superior spectroscopic characteristics of the $\mathrm{CzBTCz}$ Jdots result in two orders of magnitude brighter photoluminescence of the Jdots compared with semiconductor quantum dots, which enables continuous single-Jdots imaging over a $1 \mathrm{~h}$ period. Comparison with structurally similar CzBT nanoparticles demonstrates a critical role played by the shape of $\mathrm{CzBTCz}$ on the formation of the Jdots. Our findings open an avenue for the development of a new class of fluorescent nanoparticles based on J-aggregates.

KEYWORDS: Fluorescent nanoparticles, J-aggregates, enhanced light absorption, excited-state engineering, single-particle imaging, donor-acceptor-donor-type molecule

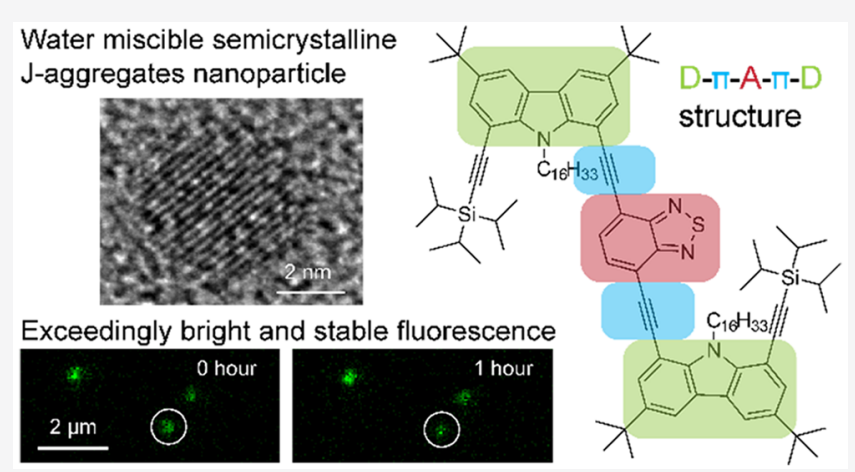

A dvanced fluorescence imaging techniques have enabled visualization and characterization of biological structures and phenomena in unprecedented spatiotemporal resolution. ${ }^{1}$ Currently, development of new fluorescent probes is one of the major focus areas for the further advancement of state-of-theart microscopy techniques. ${ }^{2-5}$ General characteristics of an ideal fluorescent probe include large fluorescence quantum yield $\left(\phi_{\mathrm{fl}}\right)$, large molar extinction coefficient $(\varepsilon)$, large twophoton absorption cross section $(\delta)$, narrow spectral bandwidth, water solubility, bio- and photostability, low cytotoxicity, and small size. The last decade has seen a surge in the development of new fluorescent nanomaterials that include semiconductor quantum dots (Qdots), ${ }^{6}$ conjugated polymer nanoparticles (polymer dots), ${ }^{7}$ carbon dots, ${ }^{8}$ metal nanoclusters, ${ }^{9}$ lanthanide-doped nanoparticles, ${ }^{10}$ and dyedoped nanoparticles. ${ }^{11}$

Among others, organic molecules are a primary choice of the fluorophore in the vast majority of experimental settings. By harnessing their flexibility in the molecular design and synthesis, organic molecules that form nanoparticles with a bright fluorescence have been developed. A most frequently used strategy is to design molecules that maintain their fluorescence properties in an aggregate state by avoiding aggregate-induced fluorescence quenching. This has been achieved by either introducing a bulky group that prevents the molecules from $\pi-\pi$ interaction ${ }^{12}$ or restricting intra- molecular motion of conformationally flexible molecules that leads to aggregation-induced emission (AIE). ${ }^{13,14}$ Although these design concepts have been successful in the fabrication of highly fluorescent organic nanoparticles and used for imaging and sensing applications, a bright fluorescence has been achieved at the cost of high density of light-emitting moieties inside the particles as well as flexible molecular design, which is inevitable in these approaches.

An alternative and more attractive strategy is to modify fluorescence properties of organic nanoparticles through an aggregation-induced engineering of electronic states of the molecules inside the particles. A promising candidate for this approach is J-aggregates in which excited-state transition dipoles of monomer molecules in the aggregates are strongly coupled, leading to a delocalization of the excited state over a large number of monomers. ${ }^{15-17}$ This causes a significant change in spectroscopic properties of the molecules in the aggregates, including significant narrowing of both absorption

Received: December 15, 2020

Revised: $\quad$ March 23, 2021 
a

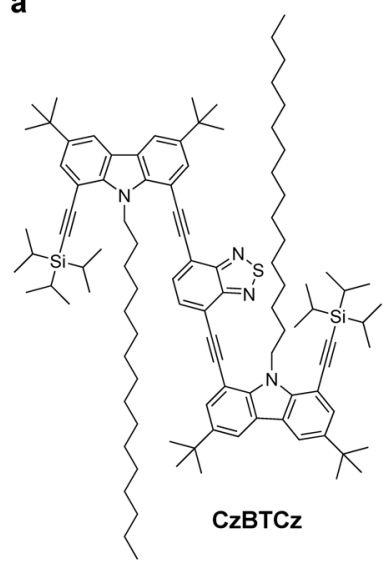

d

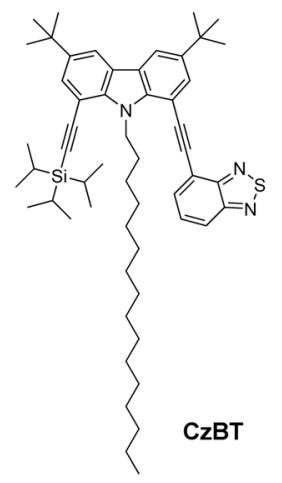

b

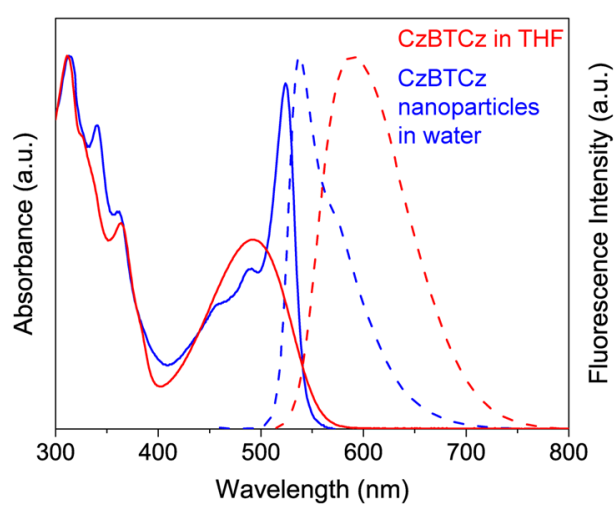

e

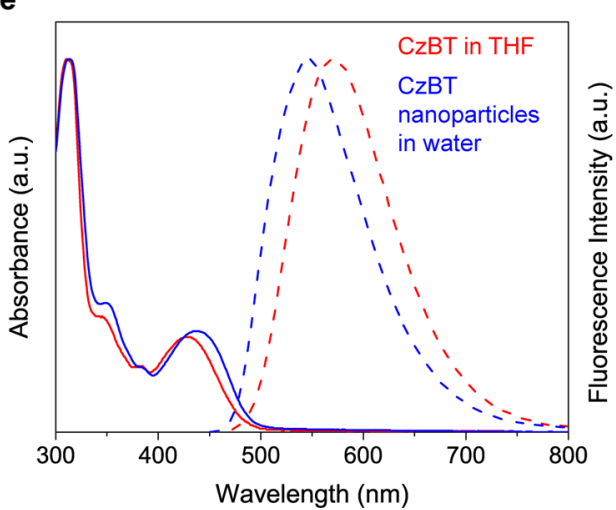

C

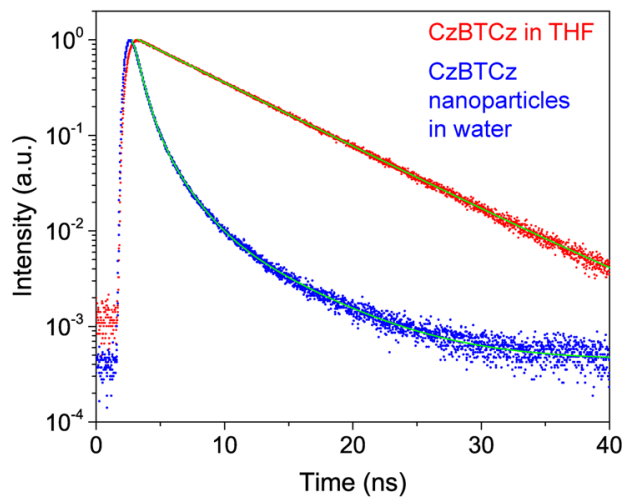

f

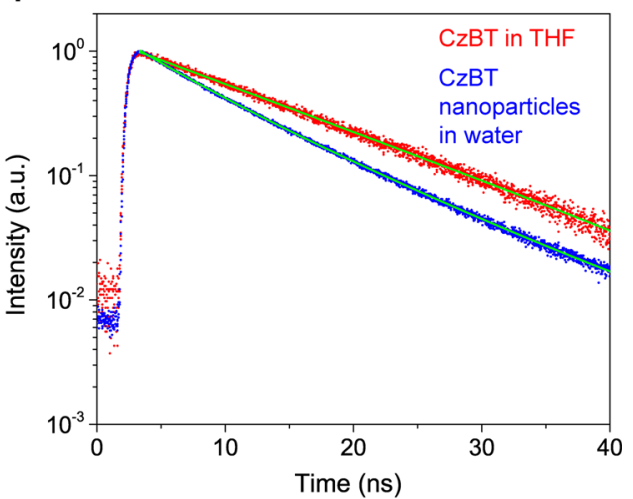

Figure 1. Spectroscopic properties of the CzBTCz Jdots. (a) Chemical structure of CzBTCz. (b) Steady-state absorption (solid lines) and fluorescence (dashed lines) spectra of $\mathrm{CzBTCz}$ in THF (red lines) and the $\mathrm{CzBTCz}$ nanoparticles dispersed in water (blue lines). The fluorescence spectra were measured upon excitation at $450 \mathrm{~nm}$. (c) Fluorescence decays obtained for $\mathrm{CzBTCz}$ in THF (red) and the CzBTCz nanoparticles dispersed in water (blue). The solid lines show fitting of the data to single- or double-exponential decaying functions. (d) Chemical structure of CzBT. (e) Steady-state absorption (solid lines) and fluorescence (dashed lines) spectra of CzBT in THF (red lines) and the CzBT nanoparticles dispersed in water (blue lines). The fluorescence spectra were measured upon excitation at $420 \mathrm{~nm}$. (f) Fluorescence decays obtained for CzBT in THF (red) and the CzBT nanoparticles dispersed in water (blue). The solid lines show fitting of the data to single- or double-exponential decaying functions.

Table 1. Spectroscopic Characteristics of CzBTCz and CzBT Nanoparticles

\begin{tabular}{|c|c|c|c|c|c|c|c|c|}
\hline & $\phi_{\mathrm{fl}}$ & $\tau_{\mathrm{fl}}(\mathrm{ns})$ & $k_{\mathrm{r}}\left(\mathrm{s}^{-1}\right)$ & $k_{\mathrm{nr}}\left(\mathrm{s}^{-1}\right)$ & $\varepsilon\left(\mathrm{M}^{-1} \mathrm{~cm}^{-1}\right)$ & $\lambda_{\mathrm{abs}}(\mathrm{nm})$ & $\lambda_{\mathrm{fl}}(\mathrm{mn})$ & size $(\mathrm{nm})^{a}$ \\
\hline $\mathrm{CzBTCz}^{b}$ & 0.97 & 6.5 & $1.5 \times 10^{8}$ & $7.7 \times 10^{6}$ & $2.4 \times 10^{4}$ & 492 & 590 & \\
\hline $\mathrm{CzBTCz}_{\mathrm{Jdots}}{ }^{c}$ & 0.95 & 1.7 & $5.7 \times 10^{8}$ & $1.8 \times 10^{7}$ & $8.1 \times 10^{6}$ & 524 & 538 & 3.5 \\
\hline $\mathrm{CzBT}^{b}$ & 0.91 & 11.4 & $8.0 \times 10^{7}$ & $7.9 \times 10^{6}$ & $9.4 \times 10^{3}$ & 428 & 570 & \\
\hline $\mathrm{CzBT}_{\mathrm{NPs}}^{c, d}$ & 0.32 & 8.7 & $3.7 \times 10^{7}$ & $7.8 \times 10^{7}$ & $3.2 \times 10^{6}$ & 438 & 546 & 14 \\
\hline$(\mathrm{CzBT})_{n}$ Pdots $^{c, e}$ & 0.16 & 2.9 & $5.5 \times 10^{7}$ & $2.9 \times 10^{8}$ & $2.7 \times 10^{6}$ & 497 & 631 & 3.0 \\
\hline
\end{tabular}

${ }^{a}$ Diameter of the particles determined by TEM. ${ }^{b}$ Measured in THF. ${ }^{c}$ Measured in water. ${ }^{d}$ Nanoparticles (NPs). ${ }^{e}$ Polymer dots (Pdots).

and fluorescence spectra, enhancement of $\varepsilon$, preservation of (or even increased) $\phi_{\mathrm{fl}}$, and decreased fluorescence lifetime $\left(\tau_{\mathrm{fl}}\right){ }^{18}$ Therefore, J-aggregates could, in principle, be an ideal fluorescent probe for fluorescence microscopy application. However, previously reported J-aggregates formed in an aqueous environment showed relatively low $\phi_{\mathrm{fl}} \cdot{ }^{19-21}$ Highly fluorescent J-aggregates have been reported only for a small number of molecules in a nonaqueous environment. ${ }^{22}$ Also, stable sub-5 nm J-aggregate nanoparticles have not been developed to date. These issues are originated mainly from the limited number of molecules that form J-aggregates. ${ }^{23-26}$

Here, we report sub-5 nm J-aggregate nanoparticles (Jdots) that display a near unity fluorescence quantum yield. Inspired by our previous study on ultrasmall donor-acceptor (D- $\pi-A)$ type polymer dots consisting of a carbazole $(\mathrm{Cz})$ donor and benzothiadiazole (BT) acceptor that demonstrated polymerchain-packing-dependent fluorescence properties, ${ }^{27,28}$ we conceived an idea of using the structural units of these polymers to fabricate nanoparticles with the expectation that we can better engineer photophysical characteristics of the molecules in an aggregation state. Surprisingly, we found that the fabricated nanoparticles exhibited the features of J-aggregates.

\section{SPECTROSCOPIC CHARACTERIZATION OF CZBTCZ NANOPARTICLES}

We fabricated nanoparticles composed of CzBTCz (D- $\pi-A-\pi$ $\mathrm{D}$ structure, Figure $1 \mathrm{a}$ ) and $\mathrm{CzBT}$ (D- $\pi$ - $A$ structure, Figure $1 \mathrm{~d}$ ) using the nanoprecipitation method (see the Materials and Methods in the Supporting Informaiton). $\mathrm{CzBTCz}$ in 
a

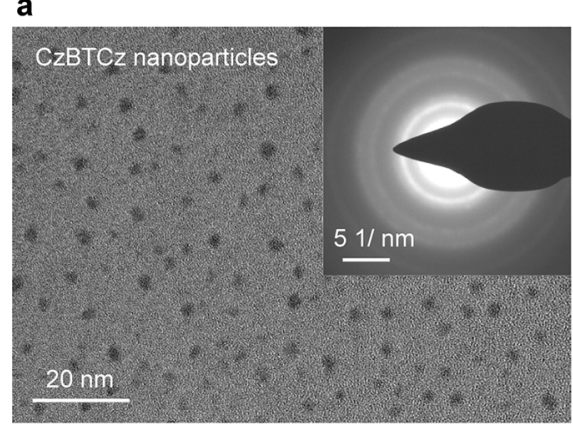

c

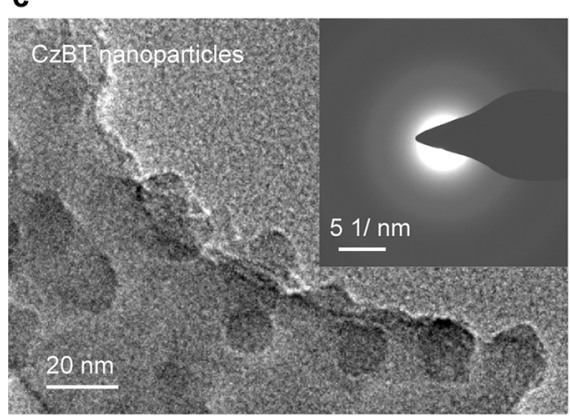

b

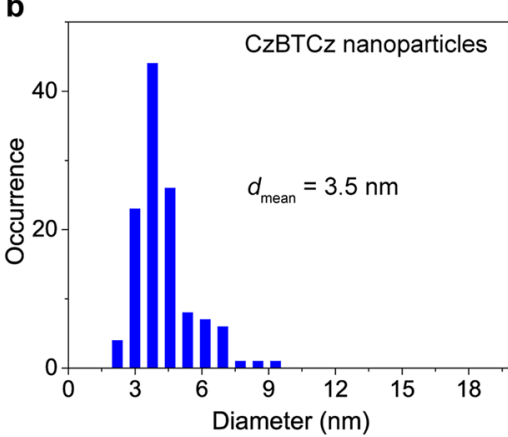

d

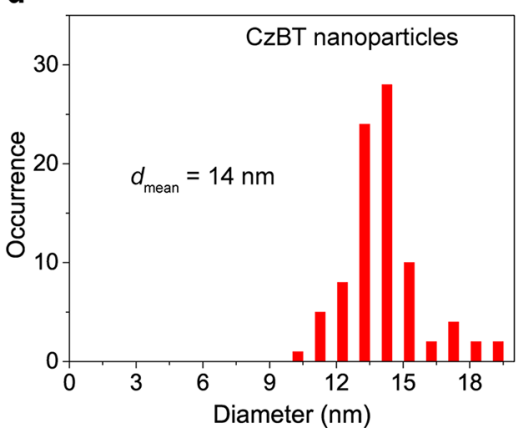

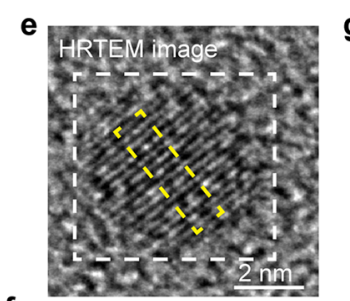

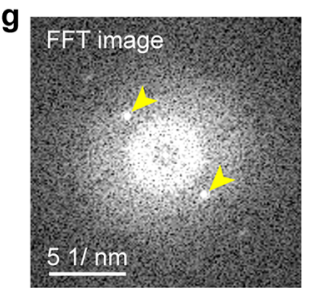

f

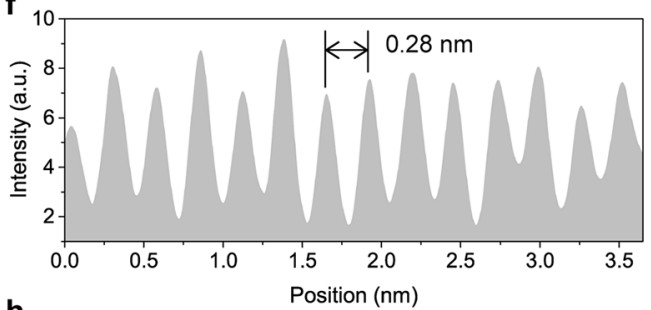

h

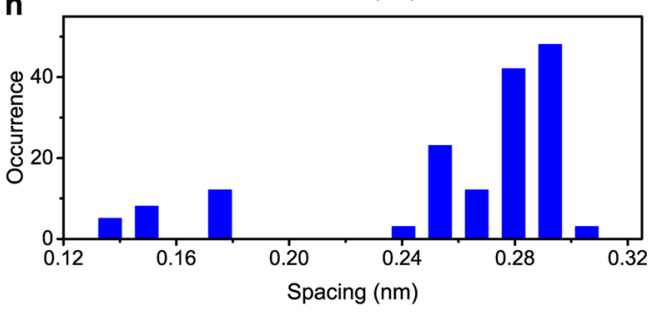

Figure 2. Structural characterization of the CzBTCz Jdots. (a) Transmission electron microscopy (TEM) image of the CzBTCz nanoparticles. The inset shows a selected area electron diffraction (SAED) pattern obtained from the particles. (b) Frequency histogram showing the size distribution of the $\mathrm{CzBTCz}$ nanoparticles. (c) TEM image of the CzBT nanoparticles. The inset shows a SAED pattern obtained from the particles. (d) Frequency histogram showing the size distribution of the CzBT nanoparticles. (e) High-resolution TEM image of a single CzBTCz nanoparticle. The yellow box shows the region from which the intensity profile displayed in (f) was calculated. The white box shows the region from which the fast Fourier transform (FFT) image displayed in (g) was calculated. The yellow arrows in (g) highlight spots in the FFT image that confirm the presence of the single-crystalline-like periodical structure in the $\mathrm{CzBTCz}$ nanoparticle displayed in (e). (h) Frequency histogram showing the distribution of the spacing observed in the $\mathrm{CzBTCz}$ nanoparticles.

tetrahydrofuran (THF) showed broad featureless absorption $\left(\right.$ fwhm $\left.=92 \mathrm{~nm}\left(3982 \mathrm{~cm}^{-1}\right)\right)$ and fluorescence $($ fwhm $=97$ $\left.\mathrm{nm}\left(2690 \mathrm{~cm}^{-1}\right)\right)$ spectra peaking at 492 and $590 \mathrm{~nm}$, respectively, typical for charge-transfer (CT) absorption and fluorescence (Figure 1b, Table 1). ${ }^{27}$ The absorption band was previously attributed to intramolecular charge-transfer (ICT) absorption between the donor $\mathrm{Cz}$ and the acceptor BT moieties. $^{29} \mathrm{CzBTCz}$ in THF exhibited a high fluorescence quantum yield $\left(\phi_{\mathrm{fl}}=0.97\right)$ with a fluorescence lifetime of 6.5 ns (Figure 1c, Table 1).

In contrast to the frequently observed ICT spectra in THF, the fabricated $\mathrm{CzBTCz}$ nanoparticles dispersed in water displayed a distinctly narrower (fwhm $=27 \mathrm{~nm}$ (1036 $\left.\mathrm{cm}^{-1}\right)$ ) and red-shifted absorption spectrum peaking at 524 $\mathrm{nm}$ (Figure 1b, Table 1). We also observed a significantly narrower fluorescence spectrum with vibronic structures peaking at $538 \mathrm{~nm}\left(\mathrm{fwhm}=55 \mathrm{~nm}\left(1800 \mathrm{~cm}^{-1}\right)\right.$, Figure $1 \mathrm{~b}$, Table 1) and a reduction of fluorescence lifetime $\left(\tau_{\mathrm{fl}}=1.7 \mathrm{~ns}\right.$, Figure 1c, Table 1) upon the particle formation (Supporting Note 1). The width of the fluorescence spectrum was much narrower than that in a nonpolar solution (Figure S1). Therefore, the observed spectral narrowing cannot be attributed to local environment-dependent spectral change. ${ }^{29}$ Interestingly, $\phi_{\mathrm{fl}}$ remains almost unchanged $\left(\phi_{\mathrm{fl}}=0.95\right.$, Table 1).

The narrow and red-shifted absorption spectrum is a signature of strong coupling and high-order stacking of $\mathrm{CzBTCz}$ inside the nanoparticles. ${ }^{15,16}$ Further, the decrease in $\tau_{\mathrm{fl}}$ observed for the $\mathrm{CzBTCz}$ nanoparticles is due to an increase in the radiative rate constant in the nanoparticles
$\left(k_{\mathrm{r}}^{\mathrm{NPs}}=5.7 \times 10^{8} \mathrm{~s}^{-1}\right)$ compared with that in THF $\left(k_{\mathrm{r}}^{\mathrm{THF}}=1.5\right.$ $\times 10^{8} \mathrm{~s}^{-1}$ ) (Table 1 ). This enhanced radiative decay rate, a phenomenon usually referred to as exciton superradiance (see Supporting Note 2), is a key characteristic of J-aggregates. ${ }^{30}$ Together, our findings strongly suggest the formation of $\mathrm{J}$ aggregates inside the $\mathrm{CzBTCz}$ nanoparticles (see Supporting Note 3 ). We emphasize that this study reports for the first time near unity $\phi_{\mathrm{fl}}$ of J-aggregates in an aqueous environment.

The $\varepsilon$ value of the $\mathrm{CzBTCz}$ nanoparticles was determined experimentally using fluorescence correlation spectroscopy $(\varepsilon$ $=8.1 \times 10^{6} \mathrm{M}^{-1} \mathrm{~cm}^{-1}$, see the Materials and Methods in the Supporting Information, Figure S2, and Supporting Note 4). This large $\varepsilon$ value is interpreted at least partly by the theoretically predicted enhanced peak $\varepsilon$ per monomer in strongly coupled J-aggregates (see Supporting Note 5). ${ }^{31,32}$ According to the theory, 3.6-fold enhancement of the peak $\varepsilon$ is expected from the narrowing of the absorption spectra upon the particle formation. Such large enhancement of the peak $\varepsilon$ is a result of the large exciton delocalization length $\left(N_{\mathrm{del}}\right.$, number of molecules in an aggregate over which electron is delocalized). Indeed, $N_{\text {del }}$ is calculated to be $N_{\text {del }}=19$ using the change in the width of the absorption spectra ${ }^{33}$ upon the Jaggregates formation inside the particles (see Supporting Note $6)$. This value is much larger than that for previously reported water-miscible J-aggregate nanoparticles. ${ }^{20}$ The $(\mathrm{CzBT})_{n}$ polymer dots ${ }^{27}$ whose monomer unit is identical to that of $\mathrm{CzBTCz}$ (Figure S3) but does not form J-aggregates inside the particles displayed three times smaller $\varepsilon$ with similar particle size (see Table 1 ). This result further suggests the enhanced $\varepsilon$ in the $\mathrm{CzBTCz}$ nanoparticles due to the intraparticle J- 

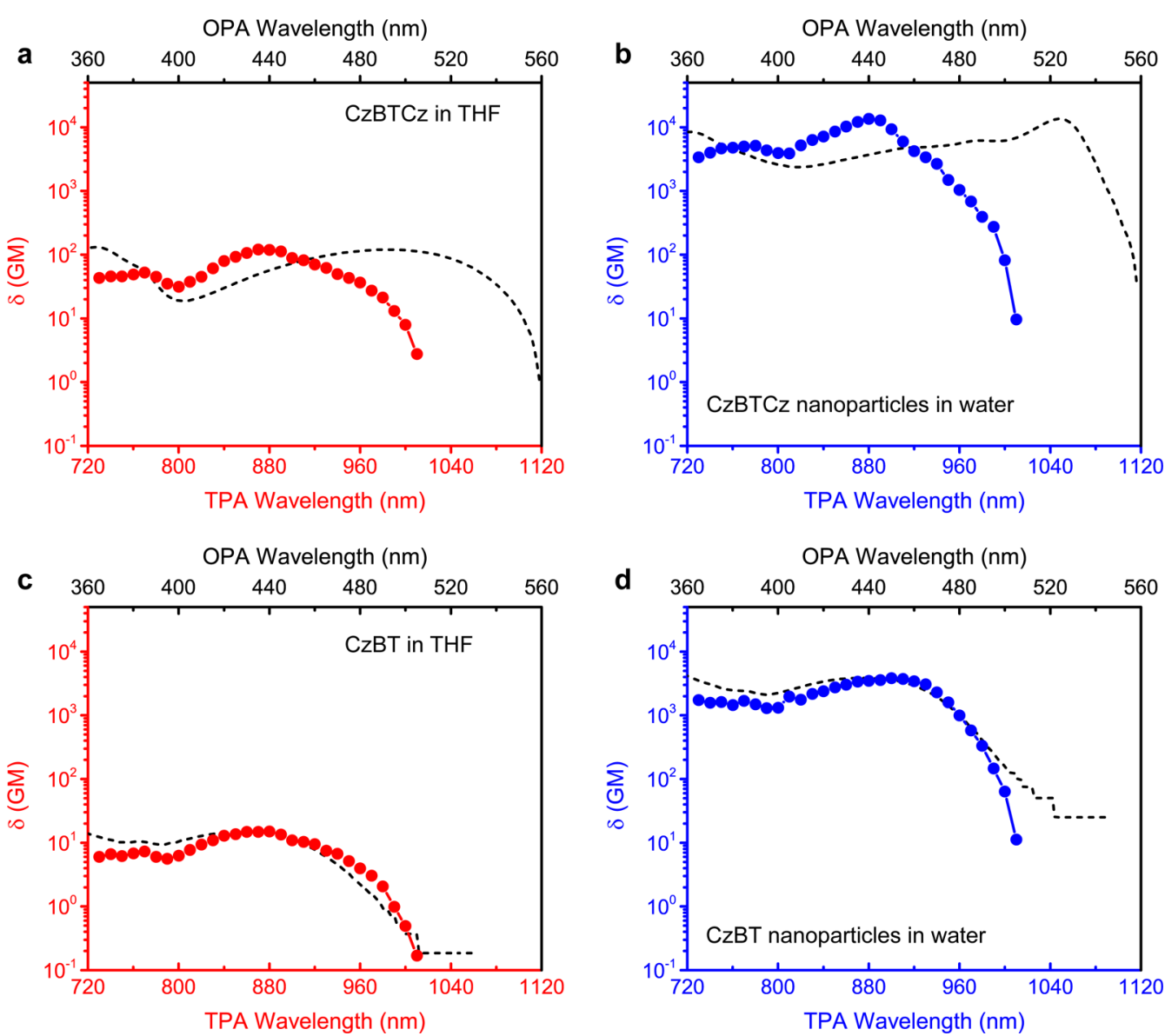

Figure 3. Two-photon absorption properties of the CzBTCz Jdots. (a) Two-photon absorption spectrum of CzBTCz in THF. The dashed line shows a one-photon absorption spectrum of CzBTCz in THF. (b) Two-photon absorption spectrum of the CzBTCz nanoparticles dispersed in water. The dashed line shows a one-photon absorption spectrum of the $\mathrm{CzBTCz}$ nanoparticles dispersed in water. (c) Two-photon absorption spectrum of CzBT in THF. The dashed line shows a one-photon absorption spectrum of CzBT in THF. (d) Two-photon absorption spectrum of the CzBT nanoparticles dispersed in water. The dashed line shows a one-photon absorption spectrum of the CzBT nanoparticles dispersed in water.

aggregates formation. In addition, the twist angle between the $\mathrm{Cz}$ and BT moiety inside the nanoparticles may contribute to the enhancement of the $\varepsilon$ value (Supporting Note 7).

In contrast to $\mathrm{CzBTCz}, \mathrm{CzBT}$ did not show any sign of $\mathrm{J}$ aggregates upon the fabrication of the nanoparticles. The CT absorption band of the CzBT nanoparticles in water peaking at $437 \mathrm{~nm}$ showed a spectral width similar to that of CzBT in THF (Figure 1e). The CzBT nanoparticles in water also displayed a broad fluorescence spectra similar to those of CzBT in THF $\left(\right.$ fwhm $_{\mathrm{NPs}}=109 \mathrm{~nm}\left(3567 \mathrm{~cm}^{-1}\right), \mathrm{fwhm}_{\mathrm{THF}}=$ $113 \mathrm{~nm}\left(3385 \mathrm{~cm}^{-1}\right)$, Figure 1e). We observed a slight decrease of the fluorescence lifetime of the CzBT nanoparticles $\left(\tau_{\mathrm{fl}}=8.7 \mathrm{~ns}\right)$ compared with that of CzBT in THF $\left(\tau_{\mathrm{fl}}=11.4\right.$ $\mathrm{ns}$, Figure 1f). This reduction in $\tau_{\mathrm{fl}}$ is mainly caused by an enhanced nonradiative decay rate $k_{\mathrm{nr}}\left(k_{\mathrm{nr}}{ }^{\mathrm{NPs}}=7.8 \times 10^{7} \mathrm{~s}^{-1}\right.$, $\left.k_{\mathrm{nr}}^{\mathrm{THF}}=7.9 \times 10^{6} \mathrm{~s}^{-1}\right)$ rather than a change in $k_{\mathrm{r}}($ see Table 1$)$. The result suggests that the fluorescence of $\mathrm{CzBT}$ is quenched upon the nanoparticle formation. Indeed, we observed a significant reduction of $\phi_{\mathrm{fl}}$ upon the particle formation $\left(\phi_{\mathrm{fl}}{ }^{\mathrm{NP}}\right.$ $=0.32, \phi_{\mathrm{fl}}^{\mathrm{THF}}=0.91$, see Table 1$)$. Together, these results demonstrate that the aggregate-induced quenching occurs inside the CzBT nanoparticles, which is frequently observed in fluorescent organic nanoparticles.

The comparison between $\mathrm{CzBT}$ and $\mathrm{CzBTCz}$ indicates that the shape of the molecule plays a key role in the formation of the J-aggregates in the $\mathrm{CzBTCz}$ nanoparticles. The overall shape of a CzBTCz molecule is similar to those of cyanine dyes that often form J-aggregates. ${ }^{34,35}$ Cyanine dyes consist of two heterocyclic rings connected by a polymethine linker. Long aliphatic chains on the heterocyclic rings contribute to efficient formation of J-aggregates. $\mathrm{CzBTCz}$ consists of two $\mathrm{Cz}$ moieties with long alkyl chains bridged by triple bonds with a BT moiety. Our findings strongly suggest that the unique spatial arrangement of the $\mathrm{Cz}$ donor and $\mathrm{BT}$ acceptor in the $\mathrm{CzBTCz}$ molecule enables the efficient formation of the J-aggregates in the nanoparticles. We would like to point out that unlike the $\mathrm{CzBTCz}$ nanoparticles, J-aggregates of cyanine dyes show much lower $\phi_{\mathrm{fl}},{ }^{36-38}$ highlighting the uniqueness of highly fluorescent $\mathrm{CzBTCz}$ J-aggregate nanoparticles (Supporting Note 8).

\section{CHARACTERIZATION OF SEMICRYSTALLINE STRUCTURE OF CZBTCZ JDOTS}

We next characterized structural properties of the $\mathrm{CzBTCz}$ nanoparticles. A transmission electron microscopy (TEM) image of the $\mathrm{CzBTCz}$ nanoparticles showed the formation of spherical-shaped nanoparticles with a relatively narrow size distribution with a mean diameter of $3.5 \mathrm{~nm}$ (Figure $2 \mathrm{a}$ and $2 \mathrm{~b}$, Supporting Note 9). A selected area electron diffraction (SAED) pattern obtained from the $\mathrm{CzBTCz}$ nanoparticles 

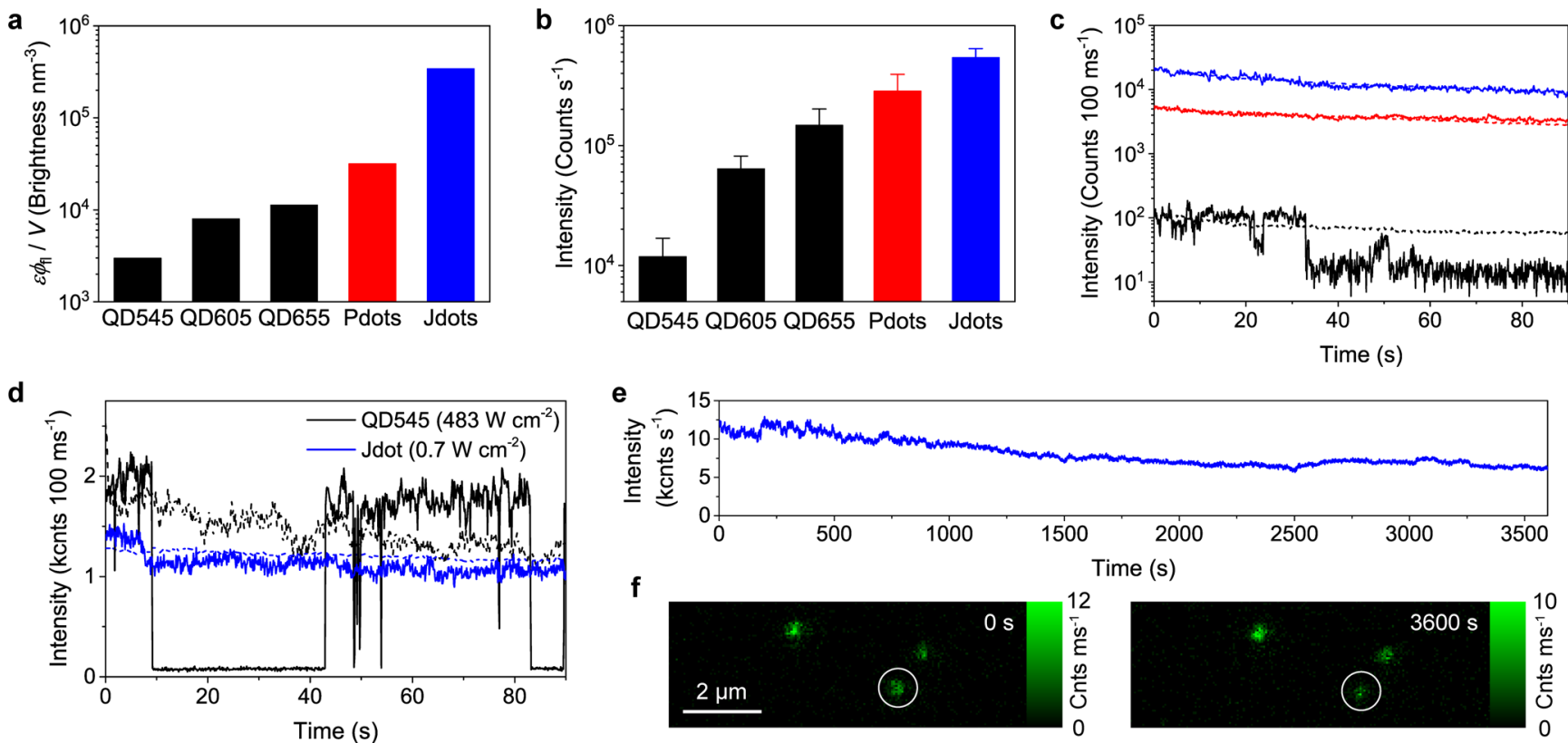

f
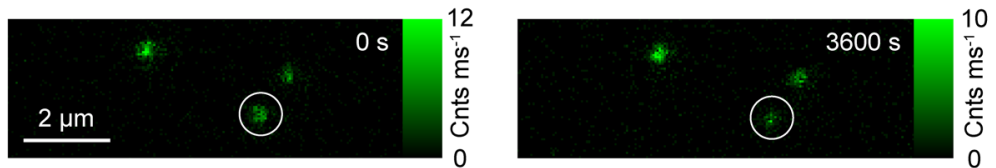

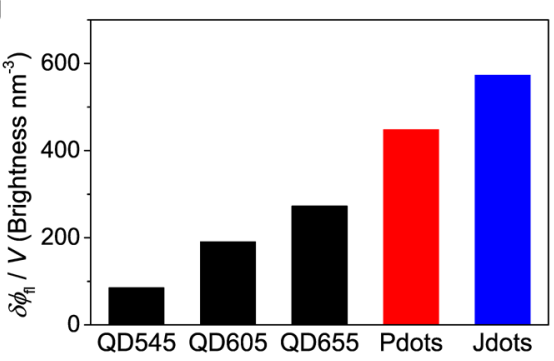

h

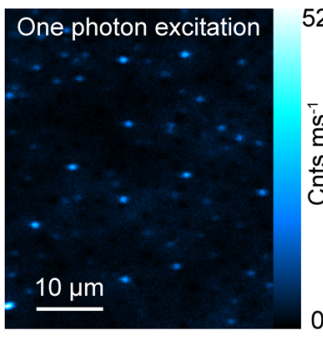

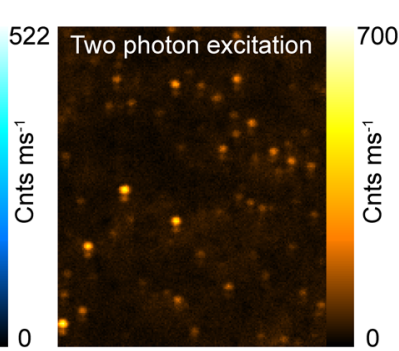

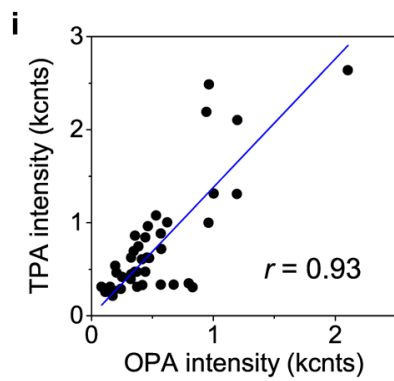

Figure 4. Fluorescence brightness of the $\mathrm{CzBTCz}$ Jdots. (a) Fluorescence brightness per unit volume calculated for Qdots 545 (QD545), Qdots 605 (QD605), Qdots 655 (QD655), (CzBT) ${ }_{n}$ polymer dots (Pdots), and CzBTCz Jdots (Jdots) under the one-photon excitation condition. (b) Mean fluorescence intensities of individual QD545, QD605, QD655, Pdots, and Jdots obtained under identical experimental conditions (485 nm excitation, $424 \mathrm{~W} \mathrm{~cm}^{-2}$ excitation power). Error bars show standard deviations determined by 1275, 586, 1663, 611, and 1871 molecules for the QD545, QD605, QD655, Pdots, and Jdots, respectively. (c) Examples of fluorescence intensity trajectories of single Qdots 545 (black line), $(\mathrm{CzBT})_{n}$ Pdots (red line), and $\mathrm{CzBTCz}$ Jdots (blue line) obtained under identical experimental conditions $\left(485 \mathrm{~nm}\right.$ excitation, $160 \mathrm{~W} \mathrm{~cm}^{-2}$ excitation power). The dashed lines show mean fluorescence intensity trajectories of 65,84 , and 115 molecules for the Qdots 545 , (CzBT) ${ }_{n}$ Pdots, and $\mathrm{CzBTCz}$ Jdots, respectively. (d) Examples of fluorescence intensity trajectories of single Qdots 545 (black line) and CzBTCz Jdots (blue line) obtained under conditions that gave similar fluorescence intensity ( $488 \mathrm{~nm}$ excitation, 483 and $0.7 \mathrm{~W} \mathrm{~cm}^{-2}$ excitation power for the Qdots 545 and $\mathrm{CzBTCz}$ Jdots). The dashed lines show mean fluorescence intensity trajectories of 108 and 60 molecules for the Qdots 545 and CzBTCz Jdots, respectively. (e) Fluorescence intensity trajectory obtained from a single $\mathrm{CzBTCz}$ Jdot over a $1 \mathrm{~h}$ period at the excitation power of $0.7 \mathrm{~W} \mathrm{~cm}^{-2}$. (f) Fluorescence images of the $\mathrm{CzBTCz}$ Jdots captured at time point $0 \mathrm{~s}$ (left) and $3600 \mathrm{~s}$ (right). White circles highlight the Jdot from which the intensity time trajectory shown in (e) was obtained. (g) Fluorescence brightness per unit volume calculated for Qdots 545, Qdots 605, Qdots 655, $(\mathrm{CzBT})_{n}$ Pdots, and $\mathrm{CzBTCz}$ Jdots under the two-photon excitation condition. (h) Fluorescence images of single CzBTCz Jdots captured under one-photon excitation (left, $488 \mathrm{~nm}$ excitation, $27 \mathrm{~W} \mathrm{~cm}^{-2}$ excitation power) and two-photon excitation (right, $880 \mathrm{~nm}$ excitation, $1.85 \mathrm{~kW} \mathrm{~cm}{ }^{-2}$ excitation power) conditions. (i) Fluorescence intensity obtained from individual CzBTCz Jdots under one- and two-photon excitation conditions. Each dot shows intensities obtained for a single Jdot by one- or two-photon excitation. The solid line shows a linear fit, and $r$ is Pearson's correlation coefficient.

clearly showed sharp rings, a typical spatial pattern observed in polycrystalline materials (Figure $2 \mathrm{a}$ inset). In contrast, the crystalline structure was not observed for the CzBT nanoparticles that have a spherical shape with a mean diameter of $14 \mathrm{~nm}$ (Figure 2c, 2d). Instead, we observed a halo-like SAED pattern from the CzBT nanoparticles, a typical spatial pattern observed from amorphous materials (Figure $2 \mathrm{c}$ inset). A highresolution (HR) TEM image of the $\mathrm{CzBTCz}$ nanoparticles clearly revealed lattice fringes with a $0.28 \mathrm{~nm}$ lattice spacing (Figure 2e, 2f). A fast Fourier transform (FFT) analysis of the image also indicates a lattice spacing of $0.28 \mathrm{~nm}$ (Figure $2 \mathrm{~g}$, 2h). While most of the $3.5 \mathrm{~nm} \mathrm{CzBTCz}$ nanoparticles showed a single crystalline domain, (Figure $2 \mathrm{e}, 2 \mathrm{f}, 2 \mathrm{~g}$ ), a small fraction of the nanoparticles, in particular larger nanoparticles, exhibited multicrystalline domains inside the particles (Figure S4).

These findings are consistent with the spectroscopic properties of the $\mathrm{CzBTCz}$ and $\mathrm{CzBT}$ nanoparticles and strongly suggest the formation of ultrasmall $3.5 \mathrm{~nm} \mathrm{CzBTCz}$ Jdots in which the $\mathrm{CzBTCz}$ molecules form strongly coupled Jaggregates. The $0.28 \mathrm{~nm}$ lattice fringe observed for the CzBTCz Jdots is similar to the previously reported spatial periodicity of other organic J-aggregates $(0.26-0.31 \mathrm{~nm}) .{ }^{39,40}$ In addition, the $0.28 \mathrm{~nm}$ spacing is smaller than the distance between $\pi-\pi$ stacked (i.e., weak coupling) triisopropylsilylethynyl pentacene molecules $(0.32-0.35 \mathrm{~nm})$, which has 
structural similarity with $\mathrm{CzBTCz}$ (i.e., flat aromatic ring connected to triisopropylsilyl groups through triple bonds). ${ }^{41}$ These results further suggest that strongly coupled J-aggregates are formed inside the $\mathrm{CzBTCz}$ Jdots. We note that we cannot rule out the possibility that graphene-like carbon nanoparticles are generated by the electron beam irradiation during the HRTEM experiments as the lattice parameters of graphene $(0.21,0.24$, and $0.34 \mathrm{~nm})$ are close to the observed $0.28 \mathrm{~nm}$ lattice spacing (Supporting Note 10). ${ }^{42}$

An origin of the observed size difference between the $\mathrm{CzBTCz}$ Jdots and the $\mathrm{CzBT}$ nanoparticles is not clear at the moment. The large zeta potential $(-45 \mathrm{mV})$ of the $\mathrm{CzBTCz}$ Jdots compared with that of the CzBT nanoparticles $(-12$ $\mathrm{mV}$ ) indicates better colloidal stability of the CzBTCz Jdots. This higher colloidal stability may contribute to the formation of the ultrasmall $3.5 \mathrm{~nm}$ size Jdots without further growth or nonspecific aggregations of the particles.

\section{TWO-PHOTON ABSORPTION OF CZBTCZ JDOTS}

We next investigated two-photon absorption (TPA)-induced fluorescence of the $\mathrm{CzBTCz}$ Jdots (Figures $\mathrm{S} 5$ and $\mathrm{S6}$ ). $\mathrm{CzBTCz}$ in THF showed a relatively large TPA cross-section $(\delta=120 \mathrm{GM}$, Figure 3a), which is 8 times larger than that of CzBT in THF ( $\delta=15$ GM, Figure $3 \mathrm{c})$. The large TPA crosssection of $\mathrm{CzBTCz}$ can be attributed to its symmetric $\mathrm{D}-\pi-A-\pi$ $\mathrm{D}$ structure, which has been known as one of the best structures for a large TPA cross-section. ${ }^{43,44}$ The TPA spectrum of $\mathrm{CzBTCz}$ in THF showed a significant blue shift compared with a one-photon absorption (OPA) spectrum of $\mathrm{CzBTCz}$ in THF (Figure 3a, Figure S7), which can be attributed to different selection rules for OPA and TPA in quadrupole molecules. $^{43,45}$ In contrast, CzBT in THF showed similar OPA and TPA spectra (Figure 3c, Figure S7), which is expected for an asymmetric $\mathrm{D}-\pi$ - $A$ type molecule.

The $\mathrm{CzBTCz}$ Jdots dispersed in water displayed a narrow TPA spectrum (fwhm $=32 \mathrm{~nm}$, Figure $3 \mathrm{~b}$, Figure S7). The width of the spectrum is similar to the OPA spectrum of the CzBTCz Jdots (fwhm $=27 \mathrm{~nm}$ ), indicating that the spectroscopic unit of the J-aggregates in the particle that are responsible for the TPA is similar to that in the OPA. However, we observed significant blue shift of the TPA spectrum of the $\mathrm{CzBTCz}$ Jdots compared with the OPA spectrum of the CzBTCz Jdots (Figure 3b, Figure S7). Indeed, the peak wavelength of the TPA spectrum of the $\mathrm{CzBTCz}$ Jdots is similar to that of $\mathrm{CzBTCz}$ in THF (Figure $3 \mathrm{a}$ and $3 \mathrm{~b}$, Figure $\mathrm{S7}$ ). This result suggests that the selection rule for the TPA of the CzBTCz is not affected significantly by the Jaggregates formation inside the particles. Due to the high packing density of the $\mathrm{CzBTCz}$ molecules in the Jdots, we observed a very large TPA cross-section of the $3.5 \mathrm{~nm}$ size CzBTCz J-dots $(\delta=13,540$ GM, Figure $3 \mathrm{~b})$. The TPA spectrum of the CzBT nanoparticles is very similar to that of $\mathrm{CzBT}$ in THF (Figure 3D), which is consistent with the results obtained from the OPA spectra and demonstrated the absence of specific intermolecular interaction inside the particles.

\section{FLUORESCENCE BRIGHTNESS OF CZBTCZ JDOTS}

The results reported in this study demonstrated that the CzBTCz Jdots have excellent characteristics as a fluorescent probe, including the narrow absorption bandwidth (fwhm $=27$ nm), J-aggregation-induced enhanced peak $\varepsilon\left(\varepsilon=8.1 \times 10^{6}\right.$ $\left.\mathrm{M}^{-1} \mathrm{~cm}^{-1}\right)$ and $\delta(\delta=13,540 \mathrm{GM})$, near unity $\phi_{\mathrm{fl}}\left(\phi_{\mathrm{fl}}=0.95\right)$, ultrasmall particle size $(d=3.5 \mathrm{~nm})$, and high stability in aqueous solution. The relationship $\varepsilon \phi_{\mathrm{fl}} / V$ (i.e., fluorescence brightness per unit volume) provides a benchmark for overall brightness of fluorescent probes, where $V$ is the volume of the probe. A calculated $\varepsilon \phi_{\mathrm{fl}} / V$ value of the CzBTCz Jdots $(3.4 \times$ $\left.10^{5}\right)$ is two orders of magnitude larger than that determined for semiconductor quantum dots (Qdots) that emit photoluminescence in the similar wavelength region (Figure $4 \mathrm{a}$, Table S1, Figure S8), mainly due to its very large $\varepsilon$ and very small size. Further, the $\varepsilon \phi_{\mathrm{f}} / V$ value is an order of magnitude larger than that of the $(\mathrm{CzBT})_{n}$ polymer dots that consist of the polymer with the same repeating unit as $\mathrm{CzBTCz}$ (Figure $4 a)$. The near unity $\phi_{\mathrm{fl}}$ of $\mathrm{CzBTCz}$ under the densely packed conditions in the particles enables the exceedingly high $\varepsilon \phi_{\mathrm{fl}} / V$ value (Supporting Note 11).

A comparison of fluorescence intensity trajectories obtained from single $\mathrm{CzBTCz}$ Jdots, $(\mathrm{CzBT})_{n}$ polymer dots, and Qdots545 at an identical data acquisition condition indeed revealed that the $\mathrm{CzBTCz}$ Jdots show much brighter fluorescence than the $(\mathrm{CzBT})_{n}$ polymer dots and Qdots 545 (Figure $4 \mathrm{~b}$ and $4 \mathrm{c}$, Supporting Note 11 ). The very large peak $\varepsilon$ due to the presence of multiple emitters inside the particles is responsible for the brighter fluorescence of the Jdots compared with the Qdots that have only a single emitter inside the particles. Further, fluorescence intensity trajectories of these fluorophores recorded at different excitation powers that result in a similar photon count rate, which is more relevant to fluorescence imaging applications, demonstrated that the CzBTCz Jdots exhibited stable fluorescence over a long period of time without displaying any fluorescence blinking behavior (Figure $4 \mathrm{~d}, 4 \mathrm{e}$ ). Even after $1 \mathrm{~h}$ of continued excitation, we were able to detect bright fluorescence from a single $\mathrm{CzBTCz}$ Jdots (Figure 4e, 4f). The CzBTCz Jdots also exhibited higher fluorescence brightness per unit volume under the two-photon excitation condition $\left(\delta \phi_{\mathrm{fl}} / V\right)$ compared with the Qdots and polymer dots (Figure $4 \mathrm{~g}$, Table S1). Due to the very large $\delta \phi_{\mathrm{fl}} /$ $V$ value, two-photon excitation fluorescence of individual $\mathrm{CzBTCz}$ Jdots was easily detected with less than $2 \mathrm{~kW} \mathrm{~cm}$ excitation power (Figure 4h, Figure S9). The fluorescence brightness of the particles under one- and two-photon excitation conditions showed a linear relationship (Figure $4 \mathrm{i}$ ), implying that both the $\varepsilon$ and $\delta$ of each $\mathrm{CzBTCz}$ Jdot are determined simply by the particle size. These results together with its ultrasmall size collectively highlight the superior characteristics of the $\mathrm{CzBTCz}$ Jdots as a fluorescent probe, in particular in single-molecule level fluorescence imaging applications.

\section{CONCLUSION}

In summary, we developed small-molecule-based highly fluorescent organic nanoparticles with unique optical properties using a reprecipitation method. The periodical pattern found in the CzBTCz Jdots demonstrates that the molecules are packed in a spatially ordered manner, consistent with the spectroscopic data that suggest the formation of J-aggregates. The absence of the J-aggregates formation in the CzBT nanoparticles consisting of the same backbone but different shape than $\mathrm{CzBTCz}$ illustrates a critical role played by the shape of the molecule on their spatial packing inside the particles. Our result suggests that the structure of $\mathrm{CzBTCz}$, which mimics the generic cyanine dye structure consisting of two large heterocyclic components connected by a $\pi$ conjugated linker, is highly relevant to the J-aggregate 
formation. The heavy-metal-free organic Jdots constitute a promising biocompatible alternative to semiconductor nanomaterials for one- and two-photon fluorescence imaging applications. Careful design and synthesis of carbazole-based symmetric $\mathrm{D}-\pi-A-\pi-\mathrm{D}$ molecules with different acceptor moieties could enable researchers to tune the fluorescence into the near-infrared (NIR) and shortwave infrared (SWIR) spectral regions, ${ }^{28,46}$ which is more desirable in biological imaging. Utilization of self-assembled J-aggregates nanostructures could solve the common problem of limited brightness of NIR/SWIR-emitting fluorescent probes.

\section{ASSOCIATED CONTENT}

\section{SI Supporting Information}

The Supporting Information is available free of charge at https://pubs.acs.org/doi/10.1021/acs.nanolett.0c04928.

Materials and methods, supporting notes S1-S11, Figures S1-S10, and Table S1 (PDF)

\section{AUTHOR INFORMATION}

\section{Corresponding Authors}

Satoshi Habuchi - King Abdullah University of Science and Technology, Biological and Environmental Science and Engineering Division, Thuwal 23955-6900, Saudi Arabia; (1) orcid.org/0000-0002-6663-2807;

Email: Satoshi.Habuchi@kaust.edu.sa

Hubert Piwoński - King Abdullah University of Science and Technology, Biological and Environmental Science and Engineering Division, Thuwal 23955-6900, Saudi Arabia; ○ orcid.org/0000-0001-8666-3945;

Email: Hubert.Piwonski@kaust.edu.sa

\section{Authors}

Shuho Nozue - King Abdullah University of Science and Technology, Biological and Environmental Science and Engineering Division, Thuwal 23955-6900, Saudi Arabia

Hiroyuki Fujita - Tokyo Institute of Technology, Department of Materials Science and Engineering, Tokyo 152-8552, Japan

Tsuyoshi Michinobu - Tokyo Institute of Technology, Department of Materials Science and Engineering, Tokyo 152-8552, Japan; 이 orcid.org/0000-0001-6948-1189

Complete contact information is available at: https://pubs.acs.org/10.1021/acs.nanolett.0c04928

\section{Author Contributions}

S.H. and H.P. conceived the project. H.P. and S.H. designed the experiments. H.P. conducted the experiments with a help of S.N. and analyzed the data. T.M. and H.F. synthesized the polymers. H.P. and S.H. wrote the manuscript. All authors discussed the results.

\section{Notes}

The authors declare no competing financial interest.

\section{ACKNOWLEDGMENTS}

This study was supported by King Abdullah University of Science and Technology (KAUST) and the KAUST Office of Sponsored Research (OSR) under award no. OSR-2016-2967CRG5.

\section{REFERENCES}

(1) Sahl, S. J.; Hell, S. W.; Jakobs, S. Fluorescence nanoscopy in cell biology. Nat. Rev. Mol. Cell Biol. 2017, 18 (11), 685-701.

(2) Lavis, L. D.; Raines, R. T. Bright Building Blocks for Chemical Biology. ACS Chem. Biol. 2014, 9 (4), 855-866.

(3) Wolfbeis, O. S. An overview of nanoparticles commonly used in fluorescent bioimaging. Chem. Soc. Rev. 2015, 44 (14), 4743-4768.

(4) Hong, G. S.; Antaris, A. L.; Dai, H. J. Near-infrared fluorophores for biomedical imaging. Nat. Biomed. Eng. 2017, 1 (1), 0010.

(5) Grimm, J. B.; English, B. P.; Chen, J. J.; Slaughter, J. P.; Zhang, Z. J.; Revyakin, A.; Patel, R.; Macklin, J. J.; Normanno, D.; Singer, R. $\mathrm{H}$.; et al. A general method to improve fluorophores for live-cell and single-molecule microscopy. Nat. Methods 2015, 12 (3), 244-250.

(6) Kairdolf, B. A.; Smith, A. M.; Stokes, T. H.; Wang, M. D.; Young, A. N.; Nie, S. M. Semiconductor Quantum Dots for Bioimaging and Biodiagnostic Applications. Annu. Rev. Anal. Chem. 2013, 6, 143-162.

(7) Wu, C. F.; Chiu, D. T. Highly Fluorescent Semiconducting Polymer Dots for Biology and Medicine. Angew. Chem., Int. Ed. 2013, 52 (11), 3086-3109.

(8) Liu, M. L.; Chen, B. B.; Li, C. M.; Huang, C. Z. Carbon dots: synthesis, formation mechanism, fluorescence origin and sensing applications. Green Chem. 2019, 21 (3), 449-471.

(9) Kang, X.; Zhu, M. Z. Tailoring the photoluminescence of atomically precise nanoclusters. Chem. Soc. Rev. 2019, 48 (8), 24222457.

(10) Garfield, D. J.; Borys, N. J.; Hamed, S. M.; Torquato, N. A.; Tajon, C. A.; Tian, B.; Shevitski, B.; Barnard, E. S.; Suh, Y. D.; Aloni, S.; et al. Enrichment of molecular antenna triplets amplifies upconverting nanoparticle emission. Nat. Photonics 2018, 12 (7), $402-407$.

(11) Trofymchuk, K.; Reisch, A.; Didier, P.; Fras, F.; Gilliot, P.; Mely, Y.; Klymchenko, A. S. Giant light-harvesting nanoantenna for single-molecule detection in ambient light. Nat. Photonics 2017, 11 (10), 657-663.

(12) Wu, J. S.; Fechtenkotter, A.; Gauss, J.; Watson, M. D.; Kastler, M.; Fechtenkotter, C.; Wagner, M.; Mullen, K. Controlled selfassembly of hexa-peri-hexabenzocoronenes in solution. J. Am. Chem. Soc. 2004, 126 (36), 11311-11321.

(13) Lin, J. C.; Zeng, X. D.; Xiao, Y. L.; Tang, L.; Nong, J. X.; Liu, Y. F.; Zhou, H.; Ding, B. B.; Xu, F. C.; Tong, H. X.; et al. Novel nearinfrared II aggregation-induced emission dots for in vivo bioimaging. Chem. Sci. 2019, 10 (4), 1219-1226.

(14) Hong, Y. N.; Lam, J. W. Y.; Tang, B. Z. Aggregation-induced emission. Chem. Soc. Rev. 2011, 40 (11), 5361-5388.

(15) Hestand, N. J.; Spano, F. C. Molecular Aggregate Photophysics beyond the Kasha Model: Novel Design Principles for Organic Materials. Acc. Chem. Res. 2017, 50 (2), 341-350.

(16) Hestand, N. J.; Spano, F. C. Expanded Theory of H- and JMolecular Aggregates: The Effects of Vibronic Coupling and Intermolecular Charge Transfer. Chem. Rev. 2018, 118 (15), 70697163.

(17) Jelley, E. E. Spectral absorption and fluorescence of dyes in the molecular state. Nature 1936, 138, 1009-1010.

(18) Wurthner, F.; Kaiser, T. E.; Saha-Möller, C. R. J-Aggregates: From Serendipitous Discovery to Supramolecular Engineering of Functional Dye Materials. Angew. Chem., Int. Ed. 2011, 50 (15), $3376-3410$

(19) Yang, C. J.; Wang, X. C.; Wang, M. F.; Xu, K. M.; Xu, C. J. Robust Colloidal Nanoparticles of Pyrrolopyrrole Cyanine JAggregates with Bright Near-Infrared Fluorescence in Aqueous Media: From Spectral Tailoring to Bioimaging Applications. Chem. Eur. J. 2017, 23 (18), 4310-4319.

(20) Xu, Z. Z.; Liao, Q.; Wu, Y. S.; Ren, W. L.; Li, W.; Liu, L. B.; Wang, S.; Gu, Z. J.; Zhang, H. L.; Fu, H. B. Water-miscible organic Jaggregate nanoparticles as efficient two-photon fluorescent nanoprobes for bio-imaging. J. Mater. Chem. 2012, 22 (34), 17737-17743.

(21) von Berlepsch, H.; Bottcher, C.; Dahne, L. Structure of Jaggregates of pseudoisocyanine dye in aqueous solution. J. Phys. Chem. B 2000, 104 (37), 8792-8799. 
(22) Kaiser, T. E.; Stepanenko, V.; Wurthner, F. Fluorescent JAggregates of Core-Substituted Perylene Bisimides: Studies on Structure-Property Relationship, Nucleation-Elongation Mechanism, and Sergeants-and-Soldiers Principle. J. Am. Chem. Soc. 2009, 131 (19), 6719-6732.

(23) Chen, Z. J.; Liu, Y.; Wagner, W.; Stepanenko, V.; Ren, X. K.; Ogi, S.; Wurthner, F. Near-IR Absorbing J-Aggregate of an Amphiphilic BF2-Azadipyrromethene Dye by Kinetic Cooperative Self-Assembly. Angew. Chem., Int. Ed. 2017, 56 (21), 5729-5733.

(24) Sun, P. F.; Wu, Q.; Sun, X. L.; Miao, H.; Deng, W. X.; Zhang, W. S.; Fan, Q. L.; Huang, W. J-Aggregate squaraine nanoparticles with bright NIR-II fluorescence for imaging guided photothermal therapy. Chem. Commun. 2018, 54 (95), 13395-13398.

(25) Maiti, N. C.; Mazumdar, S.; Periasamy, N. J- and H-aggregates of porphyrin-surfactant complexes: Time-resolved fluorescence and other spectroscopic studies. J. Phys. Chem. B 1998, 102 (9), 15281538 .

(26) Cai, K.; Xie, J. J.; Zhao, D. H. NIR J-Aggregates of Hydroazaheptacene Tetraimides. J. Am. Chem. Soc. 2014, 136 (1), $28-31$.

(27) Piwonski, H.; Michinobu, T.; Habuchi, S. Controlling Photophysical Properties of Ultrasmall Conjugated Polymer Nanoparticles Through Polymer Chain Packing. Nat. Commun. 2017, 8, 15256.

(28) Piwonski, H.; Li, W.; Wang, Y.; Michinobu, T.; Habuchi, S. Improved fluorescence and brightness of near-infrared and shortwave infrared emitting polymer dots for bioimaging applications. ACS Appl. Polym. Mater. 2020, 2 (2), 569-577.

(29) Habuchi, S.; Fujita, H.; Michinobu, T.; Vacha, M. Twist Angle Plays an Important Role in Photophysical Properties of a DonorAcceptor-Type Conjugated Polymer: A Combined Ensemble and Single-Molecule Study. J. Phys. Chem. B 2011, 115 (49), 1440414415.

(30) Spano, F. C. The Spectral Signatures of Frenkel Polarons in Hand J-Aggregates. Acc. Chem. Res. 2010, 43 (3), 429-439.

(31) Devoe, H. Optical properties of molecular aggregates. 1. classical model of electronic absorption and refraction. J. Chem. Phys. 1964, 41 (2), 393-400.

(32) McRae, E. G.; Kasha, M. Enhancement of phosphorescence ability upon aggregation of dye molecules. J. Chem. Phys. 1958, 28 (4), 721-722.

(33) Knapp, E. W. Lineshapes of molecular aggregates - exchange narrowing and intersite correlation. Chem. Phys. 1984, 85 (1), 73-82.

(34) Chen, W.; Cheng, C. A.; Cosco, E. D.; Ramakrishnan, S.; Lingg, J. G. P.; Bruns, O. T.; Zink, J. I.; Sletten, E. M. Shortwave Infrared Imaging with J-Aggregates Stabilized in Hollow Mesoporous Silica Nanoparticles. J. Am. Chem. Soc. 2019, 141 (32), 12475-12480.

(35) Bricks, J. L.; Slominskii, Y. L.; Panas, I. D.; Demchenko, A. P. Fluorescent J-aggregates of cyanine dyes: basic research and applications review. Methods Appl. Fluoresc. 2018, 6 (1), No. 012001.

(36) Horng, M. L.; Quitevis, E. L. Excited-state dynamics of polymer-bound J-aggregates. J. Phys. Chem. 1993, 97 (47), 1240812415.

(37) Guralchuk, G. Y.; Katrunov, I. K.; Grynyov, R. S.; Sorokin, A. V.; Yefimova, S. L.; Borovoy, I. A.; Malyukin, Y. V. Anomalous surfactant-induced enhancement of luminescence quantum yield of cyanine dye J-aggregates. J. Phys. Chem. C 2008, 112 (38), 1476214768 .

(38) Busse, G.; Frederichs, B.; Petrov, N. K.; Techert, S. Structure determination of thiacyanine dye J-aggregates in thin films: Comparison between spectroscopy and wide angle X-ray scattering. Phys. Chem. Chem. Phys. 2004, 6 (13), 3309-3314.

(39) Zweck, J.; Penzkofer, A. Microstructure of indocyanine green Jaggregates in aqueous solution. Chem. Phys. 2001, 269 (1-3), 399409.

(40) Li, Z.; Mukhopadhyay, S.; Jang, S. H.; Bredas, J. L.; Jen, A. K. Y. Supramolecular Assembly of Complementary Cyanine Salt JAggregates. J. Am. Chem. Soc. 2015, 137 (37), 11920-11923.
(41) Yao, Z. F.; Wang, J. Y.; Pei, J. Control of $\pi$ - $\pi$ Stacking via Crystal Engineering in Organic Conjugated Small Molecule Crystals. Cryst. Growth Des. 2018, 18 (1), 7-15.

(42) Wang, L.; Li, W. T.; Wu, B.; Li, Z.; Pan, D. Y.; Wu, M. H. Room-temperature synthesis of graphene quantum dots via electronbeam irradiation and their application in cell imaging. Chem. Eng. J. 2017, 309, 374-380.

(43) Pawlicki, M.; Collins, H. A.; Denning, R. G.; Anderson, H. L. Two-Photon Absorption and the Design of Two-Photon Dyes. Angew. Chem., Int. Ed. 2009, 48 (18), 3244-3266.

(44) Albota, M.; Beljonne, D.; Bredas, J. L.; Ehrlich, J. E.; Fu, J. Y.; Heikal, A. A.; Hess, S. E.; Kogej, T.; Levin, M. D.; Marder, S. R.; et al. Design of organic molecules with large two-photon absorption cross sections. Science 1998, 281 (5383), 1653-1656.

(45) Dereka, B.; Rosspeintner, A.; Li, Z. Q.; Liska, R.; Vauthey, E. Direct Visualization of Excited-State Symmetry Breaking Using Ultrafast Time-Resolved Infrared Spectroscopy. J. Am. Chem. Soc. 2016, 138 (13), 4643-4649.

(46) Piwonski, H.; Wang, Y.; Li, W.; Michinobu, T.; Habuchi, S. Millimeter-Deep Detection of Single Shortwave-Infrared-Emitting Polymer Dots through Turbid Media. Nano Lett. 2020, 20 (12), $8803-8810$. 